\title{
Breaking the Silence-Language and the Making of Meaning in Plants
}

\author{
Monica Gagliano and Mavra Grimonprez \\ Centre for Evolutionary Biology, School of Animal Biology, \\ University of Western Australia, Perth, Australia.
}

\section{Abstract}

Language is often considered a key feature of being human, and human linguistic behavior has been adopted as the universal template for studying the nature of language and its evolution. Yet it is not always clear what "language" actually is, and the lack of definition calls into question the notion that human language is unique because it has no equivalent in any nonhuman species. We ask whether the use of language is truly an activity, a form of behavior, which makes us so unique and unlike other species. We tackle this question by examining language from an ecological perspective and then considering language from a wider biological viewpoint, one that enables us to explore language as a meaning-making activity at the core of every form of life, including plants. We examine how innovative philosophical thinking and scientific research similarly call into question the current limits of language in describing the botanical world and human-plant dynamics. By providing an overview of the most recent empirically grounded advances in our understanding of the "language" of others, and particularly plants, we propose that the nonhuman world is not lacking in language the way we think it is. Ultimately, the overall aim is to invite the emergence of a new truly interdisciplinary dialogue to inspire novel approaches in further philosophical and scientific investigations, where language and its power are re-focused toward conceptualizing a more integrated perception of the world. Key Words: Adaptive behaviorPlant thinking-Communication-Embodied cognition.

\section{Introduction}

anguage is often said to be one of the hallmarks of being human and is thought to have emerged from the interactions of three adaptive systems, namely individual learning, cultural transmission, and biological evolution (Christiansen $\mathrm{Ct}$ Kirby, 2003). The faculty of language has provided humans with an effective tool for classifying experiences, discriminating events, and communicating what has been learned to others, thus steering adaptive adjustments to our way of being in (and relating to) the environment. There are many theories about the origins of language (Bolhuis et al., 2014); and while the truth is that we simply do not know where, when, and how it did come about (Hauser et al., 2002), the evolution of language in humans is considered to be one of the most important events in the history of life on this planet (Maynard Smith \& Szathmáry, 1995). There is, of course, no denying that language is essential to every aspect of our everyday lives. Through words, gestures, and much more, we use language to inform others of a multitude of feelings, desires, worries, questions, insights we experience in relation to the world that surrounds us. The question is whether such use of language is truly an activity, a form of behavior, which makes us so unique and unlike other species.

The answer to this question clearly depends on how we define language and the criteria for its use. According to the Oxford English Dictionary, the formal definition of language includes

a. The system of spoken or written communication used by a particular country, people, community, etc., typically consisting of words used within a regular grammatical and syntactic structure. b. The vocal sounds by which mammals and birds communicate; (in extended use) any other signals used by animals to communicate. c. A means of communicating other than by the use of words, as gesture, facial expression, etc.; non-verbal communication.

(c) The Author(s) 2015; Published by Mary Ann Liebert, Inc. This Open Access article is distributed under the terms of the Creative Commons License (http:// creativecommons.org/licenses/by/4.0), which permits unrestricted use, distribution, and reproduction in any medium, provided the original work is properly credited. 


\section{GAGLIANO AND GRIMONPREZ}

This definition readily acknowledges that nonhuman others share with our species a variety of cognitive and perceptual mechanisms that constitute "language" in its broad sense [see in-depth discussion on faculty of language in the broad sense (FLB) and in the narrow sense (FLN) and their components by Hauser et al., 2002]. This definition denotes what language enables an organism to $d o$, including both verbal and nonverbal communication, and makes no specific reference to language as a uniquely human trait. Strangely, however, an Internet search quickly reveals that it is a narrowly understood definition of language (FLN) and its tendency to assume human uniquenessconceived by virtue of the unique capacity of recursion (Hauser et al., 2002) and discrete infinity (Chomsky, 2000)-that continue being indoctrinated in schools and universities worldwide as established scientific facts. The problem is that the empirical evidence does not support the theoretical assumption. For example, recursion may extend indefinitely in principle, but its actual usage is quite limited (e.g., Karlsson, 2010; Laury \& Ono, 2010) and might even be absent in some human languages (Everett, 2005). Additionally, recursive computations are not a unique feature of human language because they are found in other capacities, such as numerical and spatial cognition, which are more broadly distributed among nonhuman species (e.g., Gentner et al., 2006; see also Arsenijević, 2008; Arsenijević \&t Hinzen, 2010).

To address the discrepancy, we make no attempt to be all-inclusive in our approach to this huge field of research on language. Our inevitably selective treatment of the topic is primarily informed by emerging empirical evidence from the field of animal and plant behavioral ecology rather than the wide variety of perspectives offered by linguistics and psychology. It is our goal, however, to engage the transdisciplinary dialogue between the psychological and ecological understanding of language-we approach this task by bridging the gap between the human and nonhuman world in two ways. On the one hand, we bring the human world closer to nature by showing that much of human language is instinctual and by virtue of its very "materiality" closer than we think to the language of nonhuman others. On the other hand, by showing the greater complexity of nonhuman communication and thus elevating it closer to human language, we attempt to bring nature closer to the human world via, ultimately, the medium of a more universal understanding of language.

\section{On the Human Superiority Complex}

Based on the common view portrayed by a narrow definition of the faculty of language (FLN), human language is creative and unpredictable, while nonhuman (generally restricted to some animal species) communication is believed to be stereotyped, instinctive, and predictable. But are we even sure that our use of language is truly unpredictable and creative? Based on linguistic evidence, Lakoff and Johnson (1980) showed that most of what we do every day and how we experience and describe the world around us is, in fact, instinctive; we think, act, and communicate with each other using a stereotyped language that is defined by our culture through a conceptual system of metaphors that we are normally not even aware of. Perhaps it is because this system of metaphors is unavoidable and typically unconscious (Lakoff, 2010) that we come to conceive of our language as spontaneous, free, unpredictable, and creative and, by logical extension, attribute each of these characteristics to our species as a whole, leading us into a trap of idolatry. Further, though there are many apparent differences between human and nonhuman language, it is sensible to question our long-standing practice of defining the essence of our humanness through the things that nonhuman others lack (Baker, 2012). When we do so, we inevitably use the qualities and conditions of being nonhuman as oppositional foils. At best we fail to arrive at certain conclusions about our distinctiveness, and at worst we engender distorted beliefs about our own nature as well as that of nonhuman others-beliefs that then prove disastrous for our relationship both with our own natures and with those of others.

Because of its traditional foundation in human psychology and linguistics, the study of language assumes, to a greater or lesser extent, that human linguistic behavior constitutes the standard template for theorizing the issue. Clearly, taking human language as the diagnostic reference point to investigate what linguistic abilities are present in nonhuman others is inescapably anthropocentric and confines the interpretation of the communicative capacities of nonhuman others to the domain of human values and perception. To solve these problems, we need to envision an empirically tractable and phylogenetically neutral account of language (see examples from the evolution of signals and communication literature, such as Maynard Smith \& Harper, 2003; Jablonka \& Lamb, 2005; Skyrms, 2010) that resists the temptation of looking for evidence of signaling systems in the nonhuman world that exhibit the various forms of signaling and communication that jointly make up human language. And even then, such a strategy is still anthropocentrically tinted, being inevitably bounded (as previously mentioned) to our conceptual system of metaphors, which by virtue of being a bundle of incomplete representations, favors one way of seeing while obscuring others (Lakoff \& Johnson, 1980).

In this essay, we will propose a slightly different approach, one that deliberately embraces the human "filter" by employing the very same concepts such as language, meaning, symbols, and culture (whose problematic character we have noted above) to then view language from a wider biological perspective in terms of what it lets 


\section{THE LANGUAGE OF PLANTS}

us or any organism do (i.e., an embodied view of language; see review by Kelly et al., 2002; Clark, 2006; Glenberg \& Gallese, 2012). By treating language as a real and perceivable feature of the whole organism-environment system, where linguistic information is used and comes to have meaning in the same basic way as in perceptualaction situations (see Wilson \& Golonka, 2013), we are able to consider language as a meaning-making activity at the core of every form of life, whether human or not.

Here, we offer an overview of the most recent empirically grounded advances in our understanding of the actual "gestures" and "utterances" of others, and particularly plants, which may assist in opening up a fresh dialogue about and with these nonhuman others. Ultimately, the overall aim is to encourage the emergence of new ways of understanding our experience of the world and, in so doing, breathing life into a new narrative, where language is unbridled from human incarceration and its power is re-focused toward a more integrated perception of the world.

\section{The Making of Meaning}

Humans use language to make sense of everything that surrounds them and to share their perceptions with others in order to reduce uncertainty about the world and possibly improve their capacity for survival. While our drive for language acquisition may be innate (Chomsky, 1965; but see Tomasello, 2003), its competent use in relation to a given cultural background is a meaning-making activity that is acquired through learning and inevitably bound to the context of its use (i.e., language is "meaning in use" à la Wittgenstein; see Baker, 2012). In this sense, our language is about information, certainty, and survival; but most profoundly, language serves us in our attempt to grasp the essence of what it is like to experience being human.

As humans, bound as we are to our dwelling-world (see the notion of "Umwelt"; von Uexküll, 2010), is it even possible to conceive the making of meanings by others-perceive the language of a humpback whale, a songbird, an ant, a sunflower, or a bacteria? Doing so could be a matter of attunement to the enormous vocabulary of bodily postures, elusive gestures, loud colorful displays, fleeting acoustic and chemical utterances, and barely palpable electromagnetic embraces used by all living forms, including us. Of course, this is no easy task. To start with, because of our innate anthropocentric bias (see discussion by Gagliano, 2015), we are likely to recognize more readily (if at all) the existence of a language when this is primarily made of sounds (i.e., speech as the defining ability of using language to engage in dialogue and its absence used to denote the "silence" of nature; Vogel, 2006). Yet this could be just the place where we start considering the language of nonhumans from a wider biological perspective based on available empirical evidences.

Birds, for example, have a considerable repertoire of sounds, which develops into articulate and complex "songs" constituting part of their language and exists alongside their innate call system (equivalent to our human innate call system of screams, grunts, sighs, laughter). The avian language arises through learning earlier in life, and it is fully developed by experience within the context of its use, so that birds know when to use certain vocalizations (e.g., a flight whistle is used only while the birds are flying; Rothstein $\mathrm{Ct}$ Fleischer, 1987). The exquisite range of vocalizations including a variety of distinct regional dialects that makes up the avian language is motivated by a need to communicate and imbued with meanings, such as declaring ownership of a territory, attracting the attention of a potential mate, or repelling an intruder, all of which are examples of interactive exchanges between two or more individuals (i.e., conversations). Moreover, a recent study by Flower, Gribble, and Ridley found that a species of African bird is in fact rather talented at using language, especially that of other species, to "cry wolf" in a bid to scare other animals away and steal their food. As true tacticians, these birds change their calls in response to the feedback they receive from their dupes, so that when one false alarm no longer works, the birds switch to another species' warning cry (Flower et al., 2014). Whether or not these birds are actually intuiting what others are thinking or adapting their behavior by reflecting upon their meaning-making activity, the fact remains that their mastery of language is, like our own, a demonstration of a very accomplished capacity for creating sounds that acquire meaning because of how the information is used (i.e., to direct and regulate the behaviors of themselves or others), which ultimately enhances their survival.

Exactly how, though, do we approach others whose languages we fail to notice because they appear silent to us? Plants seem to fall into this particular category because theirs is primarily (as currently understood) a silent language of shapes, colors, and scents (but see recent findings on the ability of plants to produce, detect, and respond to sound; Appel \&t Cocroft, 2014; Gagliano, 2013; Gagliano et al., 2012). Indeed, one of the primary ways through which plants interact and communicate within their environment is by a rich and complex bouquet of several thousand volatile chemicals (e.g., Raguso, 2008). Through their crafty use of this chemical language, plants are able to breathe out their message by encoding it with a single scented word that nonetheless conveys multiple meanings depending on the intended recipients. By adding just a bitter whisper (literally, only tiny amounts), of nicotine to their bouquet 


\section{GAGLIANO AND GRIMONPREZ}

of nectar volatiles, for example, plants are able to discourage unwanted visitors such as florivores and nectar thieves; yet this same nectar constituent is simultaneously used by the plant to manipulate the behavior of desirable floral visitors, such as hummingbirds, causing them to visit more flowers and ultimately increasing the plant's reproductive success (Kessler \&t Baldwin, 2006; Kessler et al., 2008). Together with olfactory signals, visual displays including species-specific shapes, colors, and color patterns (see Raguso, 2004) are the other prominent and well-known medium through which plants interact and communicate. More than 450 species of plants, for example, are able to change their color, position, and shape to advertise their trading hours and even promote further business deals if inadequately pollinated. Research by Willmer, Stanley, Steijven, Matthews, and Nuttman (2009) describes the legume Desmodium setigerum as one such master shape-shifter, who will show off attractive lilac flowers at the start of the day to then turn them into a less eye-catching white and turquoise color as the flowers get pollinated over the course of the day. The color change is accompanied by a slower change in the shape of the flower as the upper petal folds downward over the reproductive parts, where the pollen is produced (i.e., anthers) and germinates (i.e., stigma). By rapidly changing the shape and color of its pollinated flowers and thus reducing their attractiveness, this plant directs pollinators to the unvisited flowers to be pollinated. If they received insufficient pollen, however, the flowers of this plant are unique in their ability to (literally) re-advertise themselves as "open for business" by changing their shape to expose the stigma once again as well as shifting from the white and turquoise back to the attentiongrabbing lilac color.

Like all language, these are gestures enriched with meaning, which plants use to invite, for example, the pollinators' behavior that is most beneficial at a specific time. Plants and pollinators, in particular, seem to have reached a mutual understanding of this interactive language, a kind of covenant where the meaning of colors, shapes, and scents together with the rules that govern these meanings are constantly negotiated, agreed upon, and embodied by the plant and its pollinators as part of their coevolutionary dialogue. In this sense, they do not differ considerably from any human gestures, including our spoken words; these, too, are perceptible material symbols "representing" the real things (or referents) of the world and to which a meaning is assigned and collectively agreed upon for a message to be shared among individuals. At this point, some may concede that plants display the faculty of language, but nonetheless argue for human exceptionalism-our language is unique and distinctive because of the arbitrariness of the relationship between its signifiers and their referents (i.e., Saussure's theory of arbitrariness; Harris, 1987), an arbitrariness that implies freedom and creativity. In other words, human language is special because of its power of abstraction, thanks to which the symbols we use are, in fact, not directly tied down to the physical things they symbolize. Yet once again, how sure are we that our language truly is as subjective and not intrinsically connected to what it represents as we perceive? Even excluding the case of onomatopoeia and ideogramic languages (discussion on this topic by Cuddon, 2013), linguistic evidence shows that our words were originally much more rooted in a material connection to the things they referred to than we think, and abstraction of the symbol from the symbolized only developed over time (see Abram, 1996, and notes therein; pp. 99-102/284).

Interestingly, even if we were to consider abstraction as an intrinsic and "required" property of human language, which allows a speaker to refer to something that is not present (as proposed by Vogel, 2006), our exceptionalism remains illusionary. After all, if the case of human hunters mimicking partridge calls to lure the birds out of the bush is an example of abstraction (Vogel, 2006), then the case mentioned above of the African bird that cries wolf using other species' warning calls is, too. And so it is for orchids that dupe wasps into becoming unsuspecting pollinators by releasing a spectrum of chemicals very similar to what other plants emit when "crying for help" to summon predatory insects to feast on caterpillars (Brodmann et al., 2008) or even mimicking animal alarm (pheromone) calls such as those of honey bees, which are frequently captured by wasps to feed on their larvae (Brodmann et al., 2009). In other words, human language is not more exceptional than the very evolutionary process that shapes it-a natural dance by which the symbolic, yet material, gestures of all living species are perpetually moved into new and evolving habits of rendering meaning in the world.

\section{The Faculty of Language: Learning, Culture, and Evolution in Plants}

The versatility in the linguistic behavior of plants, like in other organisms, arises as a result of experience and, in turn, underpins the adaptive changes necessary to cope with novel and unpredictable challenges to ensure the survival and flourishing of individuals within their specific ecological, as well as social, settings. We now know that plant chemical language, for example, is endowed with true semantic flexibility, so that new meanings may be assigned to old chemical words and used in novel interactions and new contexts (see review by Holopainen, 2004). Evolutionarily speaking, it is precisely through use and experience under different circumstances 


\section{THE LANGUAGE OF PLANTS}

that this inventory of gestures and utterances is enriched with meaning and, most importantly, shared with others and across generations. From this perspective, the language of plants seems to emerge from the interactions of individual learning, cultural transmission, and biological evolution-the same three adaptive systems thought to be at the root of human language. Let us briefly consider how these three systems are expressed in the life of plants.

Even though, in the broadest sense, the evolution of language occurs at a species level over hundreds of thousands of years, the fundamental process of adaptation by which a linguistic behavior is acquired in the first place, performed by a number of individuals over time, and then passed from one generation to the next (i.e., on a cultural timescale of thousands of years), is driven by the individual's ability to learn during its lifetime (see discussion by Christiansen and Kirby, 2003). In this regard, we have recently discovered that plants, like animals, can learn rapidly by acquiring novel behavioral features and change their behavior as a result of their individual experience (Gagliano et al., 2014); these learned behaviors can later become innate and potentially enhance evolutionary learning at the species level through the fundamental mechanism of genetic assimilation (i.e., the "Baldwin Effect"). Based on what we currently know, it is in the context of plant defensive behavior against enemies that the linguistic ability of a plant to swiftly learn appears to be particularly significant. The volatile words plants whisper, for example, appear to be rather specific in what they convey (e.g., expressing useful and reliable information to the predators of their attackers). Moreover, by remembering past attacks, individual plants learn to adjust their volatile responses to be better prepared for future battles. And, as in humans, plants eavesdrop on the affairs of their neighbors and are able to use this vicariously acquired knowledge to mount a tactical defensive response even before they are attacked or damaged themselves (e.g., Heil \&t Ton, 2008; Karban et al., 2000; Paré \&t Tumlinson, 1999). Additionally, we know that the scented utterances of plants are more meaningful and effectively received among kin (i.e., genetically identical or related) than strangers (e.g., Karban et al., 2013). This finding together with the recent discovery that recognition of relatives also involves specific leaf gestures and the perception of particular light signals (e.g., Crespi \& Casal, 2015) has demonstrated the occurrence of cooperative rather than competitive interactions among kin in plants (e.g., Dudley \& File, 2007; File et al., 2012) as observed in a wide range of taxa. And, in general, this seems analogous to the process by which a group of individuals learns and passes on information to others, hence giving rise to what we call cultural transmission in humans and some animals.
Ultimately (and evolutionarily), being able to communicate with each other, form bonds of various kinds, and engage in teamwork are what makes language so important to us all, human and nonhuman alike. Consequently, we would like to invite the reader one last time in this essay to interrogate the cultural validation of human linguistic uniqueness, in which humans strike us as "special" or "exceptional" when compared with nonhuman others. Whether human or not, the linguistic code of gestures and utterances unique to each species is designed as it is because those (and not any others) are the specific design features that have evolved to make for a useful communication system in the cultural context. Here, the term "culture" relates to the suite of behaviors and ways of living for that species or group of species. For example, the cultural background of plants refers to the interactions and relationships among plants as well as between plants, other organisms, and the environment. Therefore, the interpretation of these linguistic features is contingent on the knowledge of the appropriate cultural background in which the code is used. As such knowledge is understood and shared by members of a specific group (whether human or nonhuman), the code concurrently defines the communication system within that group and constructs the identity of those that use it. In humans, we can readily appreciate the extent to which speaking a language with a particular accent, for example, can easily give away one's geographical area of origin or even social status. And we appreciate that this is the case in other species, too. The song patterns of humpback whales, for a wellknown example, depend on where they live; populations inhabiting different ocean basins are known to sing distinctly different songs. All males in a given population sing the same mating song until a catchier tune emerges as they encounter a migrating population; then all males start singing the latest song (Garland et al., 2011; Noad et al., 2000). The degree of creativity associated with the composition of these songs and their sharing, as part of a cultural exchange among populations, is undeniable even if we still do not understand exactly how they are composed or what they communicate.

To understand this "whale-specific" knowledge, there is no need to break into the "whale code" by translating it into human standards; it is essential, however, to appreciate the cultural background within which such "whale code" is used by whales. Somehow, we intuitively know that what whales are sharing using their language is solidly reposed within the nature of being a whale. And we believe this is exactly where the crux of the matter is rooted. The answer to our earlier question of whether it is possible for humans to conceive and appreciate the making of meanings by others may also be found here. What if language is a fundamentally natural and inevitable consequence of being that 


\section{GAGLIANO AND GRIMONPREZ}

emerges as an organism makes meaning of its surroundings and, in turn, engraves the very identity of that organism and its physical embodiment in its world?

If we are to understand the language of plants, for example, the detecting, dissecting, and deciphering of their "code" (e.g., the identity of the chemicals a plant produces) need not be the main priority of our scientific endeavors. For example, how many more chemical compounds or light wavelengths do we need to detect and analyze before we can truly access, understand, and appreciate plant language? Much of the research in plant science is primarily focused on dissecting plants and deciphering their internal codes. However, the greatest advances in our understanding of plant language have been ingeniously delivered by a relatively small number of scientists who have adopted ecologically driven approaches, where the "cultural background" is taken into account. This is because meaning emerges during interactions among organisms; hence language is not a fixed property of that organism (e.g., a specific chemical compound) but rather a truly ecological, dynamic process of relationships by which meaning emerges to shape the production of behaviors that, in turn, shape new interactions for new meaning to emerge (e.g., a biology of language à la Maturana; see discussion on the topic by Kravchenko, 2011). In practice, reducing such an active and ecologically vibrant process to a fixed and Petri dish-like property has resulted in substituting the subjective material plant with an abstraction, the scientific idea of what an objectified plant is, does, and knows. Far from being trivial, the cultural construction of plants as objects of scientific exploration not only contradicts the emerging and expanded understanding of plant behavior, including matters of plant intelligence, agency, and intersubjectivity, but is also of ethical significance in the context of humanplant relations (Marder, 2013; Pelizzon \& Gagliano, in press). We suggest that an embodied conception of language could offer a valuable step toward the de-objectification of plants and the recognition of their subjectivity and inherent worth and dignity, renewing a sense of ecological intimacy and kinship with these nonhuman living others and thus promoting human care for nature (see Clayton \& Meyer, 2015).

Additionally, the task of redefining language through an embodied analysis could also be valuable to address the abstraction of our own species from nature. In fact, the same power of abstraction that has reduced living plants into lifeless objects has over time (literally) uprooted our own species from the physical connection we have to what we refer to. Because the symbols we use have become themselves separate from (and only arbitrarily related to) what those symbols symbolize, the idea that Homo sapiens is separate from and dominating over all other species (hence special) is incarnated within the medium of communication itself but nevertheless groundless. From this perspective, our abstractive power has resulted in the silencing (rather than the revealing) of the expression and faculties of ourselves as well as others, such as plants. Breaking this silence calls for a truly "cross-cultural" dialogue-a full immersion into the ecological context and beingness of humans, plants, as well as other nonhuman organisms with whom we share a common world. Such approach demands a new and enlarged sense of "cultural awareness" that appreciates both humans and plants (and any other organism) as embodied agents and recognizes that language always is contingent on the encounter of individual and subjective perceptual worlds and emerges at those points of contact and interaction in a truly ecological sense.

In today's globalized world, we are increasingly dwelling in highly cross-cultural environments; and an attitude of tolerance, respect, and appreciation for diversity is clearly fundamental for human societies to flourish in coexistence with all others (and sadly, we know too well what happens when such an attitude is not adopted). From this perspective, language is that open and tangible invitation to (literally) stop and smell the roses-as those plant volatile chemicals enter your nose, the cross-cultural dialogue is opened.

\section{Acknowledgments}

Special thanks to Ken Burak for helping me bring these ideas into focus and John Ryan, Patricia Vieira, and anonymous reviewers for insightful comments on an earlier version of the manuscript. This work was funded by an Australian Research Council Discovery Early Career Researcher Award and Early Career Fellowship Support Program of the University of Western Australia to Monica Gagliano.

\section{Author Disclosure Statement}

No competing financial interests exist.

\section{REFERENCES}

Abram, D. (1996). The spell of the sensuous. New York: Random House.

Appel, H. M., \& Cocroft, R. B. (2014). Plants respond to leaf vibrations caused by insect herbivore chewing. Oecologia, 175, 1257-1266.

Arsenijević, B. (2008). From spatial cognition to language. Biolinguistics, 2, 3-23.

Arsenijević, B., \& Hinzen, W. (2010). Recursion as a human universal and as a primitive. Biolinguistics, 4, 165-173.

Baker, N. E. (2012) The difficulty of language: Wittgenstein on animals and humans. In N. Forsberg, M. Burley \& N. Hamalainen (Eds.), Language, ethics and animal life (pp. 45-64). New York: Bloomsbury. 
THE LANGUAGE OF PLANTS

Bolhuis, J. J., Tattersall, I., Chomsky, N., \&t Berwick, R. C. (2014). How could language have evolved? PLoS Biology, 12, doi:10.1371/journal.pbio.1001934.

Brodmann, J., Twele, R., Francke, W., Hölzler, G., Zhang, Q.-H., \& Ayasse, M. (2008). Orchids mimic green-leaf volatiles to attract prey-hunting wasps for pollination. Current Biology, 18, 740-744.

Brodmann, J., Twele, R., Francke, W., Yi-bo, L., Xi-qiang, S., \&t Ayasse, M. (2009). Orchid mimics honey bee alarm pheromone in order to attract hornets for pollination. Current Biology, 19, 1368-1372.

Chomsky, N. (1965). Aspects of the theory of syntax. Cambridge, MA: MIT Press.

Chomsky, N. (2000). New horizons in the study of language and mind. Cambridge, UK: Cambridge University Press.

Christiansen, H. M., \& Kirby, S. (2003). Language evolution: Consensus and controversies. Trends in Cognitive Science, 7, 300-307.

Clark, A. (2006). Language, embodiment, and the cognitive niche. Trends in Cognitive Science, 10, 370-374.

Clayton, S., \& Meyer, G. (2015). Conservation psychology: Understanding and promoting human care for nature. Chichester, UK: Wiley-Blackwell.

Crespi, M. A., \& Casal, J. J. (2015). Photoreceptor-mediated kin recognition in plants. New Phytologist, 205, 329-338.

Cuddon, J. A. (2013). A dictionary of literary terms and literary theory. Hoboken, NJ: Blackwell Reference Online, Blackwell Publishing.

Dudley, S. A., \&t File, A. L. (2007). Kin recognition in an annual plant. Biology Letters, $3,435-438$.

Everett, D. L. (2005). Cultural constraints on grammar and cognition in Pirahã: Another look at the design features of human language. Current Anthropology, $76,621-646$

File, A. L., Murphy, G. P., \& Dudley, S. A. (2012). Fitness consequences of plants growing with siblings: Reconciling kin selection, niche partitioning and competitive ability. Proceedings of the Royal Society B, 279, 209-218.

Flower, T. P., Gribble, M., \&t Ridley, A. R. (2014). Deception by flexible alarm mimicry in an African bird. Science, 344, 513-516.

Gagliano, M. (2013). Green symphonies: A call for studies on acoustic communication in plants. Behavioural Ecology, 24, 789-796.

Gagliano, M. (2015). In a green frame of mind: Perspectives on the behavioural ecology and cognitive nature of plants. AoB Plants, 7, doi:10.1093/aobpla/ plu075.

Gagliano, M., Mancuso, S., \& Robert, D. (2012). Towards understanding plant bioacoustics. Trends in Plant Science, 17, 323-325.

Gagliano, M., Renton, M., Depczynski, M., \& Mancuso, S. (2014). Experience teaches plants to learn faster and forget slower in environments where it matters. Oecologia, 175, 63-72.

Garland, E. C., Goldisen, A. W., Rekdahl, M. L., Costantine, R., Garrigue, C., Hauser, N. D.,... Noad, M. J. (2011). Dynamic horizontal cultural transmission of humpback whale song at the ocean basin scale. Current Biology, 21, 687-691.

Gentner, T. Q., Fenn, K. M., Margoliash, D., \&t Nusbaum, H. C. (2006). Recursive syntactic pattern learning by songbirds. Nature, 440, 1204-1207.

Glenberg, A. M., \& Gallese, V. (2012). Action-based language: A theory of language acquisition, comprehension, and production. Cortex, 48, 905-922.

Harris, R. (1987). Reading Saussure: A critical commentary on the Cours de Linguistique Générale. La Salle, IL: Open Court.

Hauser, D. M., Chomsky, N., \&t Tecumseh Fitch, W. (2002). The faculty of language: What is it, who has it, and how did it evolve? Science, 298, 1569-1579.
Heil, M., \& Ton, J. (2008). Long-distance signalling in plant defence. Trends in Plant Science, 13, 264-272.

Holopainen, J. K. (2004). Multiple functions of inducible plant volatiles. Trends in Plant Science, 9, 529-533.

Jablonka, E., \& Lamb, M. J. (2005). Evolution in four dimensions: Genetic, epigenetic, behavioral, and symbolic variation in the history of life. Cambridge, MA: MIT Press.

Karban, R., Baldwin, I. T., Baxter, K. J., Laue, G., \&t Felton, G. W. (2000). Communication between plants: Induced resistance in wild tobacco plants following clipping of neighboring sagebrush. Oecologia, 125, 66-71.

Karban, R., Shiojiri, K., Ishizaki, S., Wetzel, W. C., \&t Evans, R. Y. (2013). Kin recognition affects plant communication and defence. Proceedings of the Royal Society B, 280, doi:10.1098/rspb.2012.3062.

Karlsson, F. (2010). Recursion and iteration. In H. van der Hulst (Ed.), Recursion and human language (pp. 43-67). Berlin: De Gruyter Mouton.

Kelly, S. D., Iverson, J., Terranova, J., Niego, J., Hopkins, M., \& Goldsmith, L. (2002). Putting language back in the body: Speech and gesture on three timeframes. Developmental Neuropsychology, 22, 323-349.

Kessler, D., \& Baldwin, I. T. (2006). Making sense of nectar scents: The effects of nectar secondary metabolites on floral visitors of Nicotiana attenuata. The Plant Journal, 49, 840-854.

Kessler, D., Gase, K., \& Baldwin, I. T. (2008). Field experiments with transformed plants reveal the sense of floral scents. Science, 321, 1200-1202.

Kravchenko, A. (2011). How Humberto Maturana's biology of cognition can revive the language sciences. Constructivist Foundations, 6, 352-362.

Lakoff, G. (2010). Why it matters how we frame the environment? Environmental Communication, 4, 70-81.

Lakoff, G., \& Johnson, M. (1980). Metaphors we live by. Chicago, IL: University of Chicago Press.

Language [Def. 1]. (n.d.). In Oxford English Dictionary Online, Retrieved July 15, 2015, from http://www.oed.com/view/Entry/105582?rskey=sTZcoD\&result=1.

Laury, R., \& Ono, T. (2010). Recursion in conversation: What speakers of Finnish and Japanese know how to do. In H. van der Hulst (Ed.), Recursion and human language (pp. 69-92). Berlin: De Gruyter Mouton.

Marder, M. (2013). Plant-thinking: A philosophy of vegetal life. New York: Columbia University Press.

Maynard Smith, J., \&t Harper, D. (2003). Animal signals. Oxford, UK: Oxford University Press.

Maynard Smith, J., \& Szathmáry, E. (1995). The major transitions in evolution. Oxford, UK: Oxford University Press.

Noad, M. J., Cato, D. H., Bryden, M. M., Jenner, M.-N., \& Jenner, K. C. S. (2000). Cultural revolution in whale songs. Nature, 408, 537.

Paré, P. W., \& Tumlinson, J. H. (1999). Plant volatiles as a defense against insect herbivores. Plant Physiology, 121, 325-331.

Pelizzon, A., \&t Gagliano, M. (2015). The sentience of plants: Toward a new regime of plant rights, or the intersection of animal rights and rights of nature? Australian Animal Protection Law Journal, 11.

Raguso, R. A. (2004). Flowers as sensory billboards: Progress towards an integrated understanding of floral advertisement. Current Opinion in Plant Biology, 7, 434-440.

Raguso, R. A. (2008). Wake up and smell the roses-the ecology and evolution of floral scent. Annual Review in Ecology, Evolution and Systematics, 39, 549-569. 


\section{GAGLIANO AND GRIMONPREZ}

Rothstein, S. I., \& Fleischer, R. C. (1987). Vocal dialects and their possible relation to honest status signalling in the brown-headed cowbird. Condor, $89,1-23$.

Skyrms, B. (2010). Signals: Evolution, learning, and information. Oxford, UK: Oxford University Press.

Tomasello, M. (2003). Constructing a language: A usage-based theory of language acquisition. Cambridge, MA: Harvard University Press.

Vogel, S. (2006). The silence of nature. Environmental Values, 15, 145-171.

von Uexküll, J. (2010). A foray into the world of animals and humans: With a theory of meaning. Minneapolis, MN: University of Minnesota Press.

Willmer, P., Stanley, D. A., Steijven, K., Matthews, I. M., \&t Nuttman, C. V. (2009). Bidirectional flower color and shape changes allow a second opportunity for pollination. Current Biology, 19, 919-923.

Wilson, A.D., \& Golonka, S. (2013). Embodied cognition is not what you think it is. Frontiers in Psychology, 4, doi:10.3389/fpsyg.2013.00058.
Address correspondence to:

Monica Gagliano

Centre for Evolutionary Biology

School of Animal Biology

University of Western Australia

35 Stirling Hwy

Perth, WA 6009

Australia

E-mail: monica.gagliano@uwa.edu.au

Received: March 20, 2015

Accepted: August 8, 2015 